\title{
Cell Conservative Flux Recovery and A Posteriori Error Estimate of Vertex-Centered Finite Volume Methods
}

\author{
Long Chen ${ }^{1, *}$ and Ming Wang ${ }^{2}$ \\ ${ }^{1}$ Department of Mathematics, University of California at Irvine, Irvine, CA 92697, USA \\ 2 LMAM, School of Mathematical Sciences, Peking University, Beijing 100080, China
}

Received 18 May 2012; Accepted (in revised version) 8 March 2013

Available online 31 July 2013

\begin{abstract}
A cell conservative flux recovery technique is developed here for vertexcentered finite volume methods of second order elliptic equations. It is based on solving a local Neumann problem on each control volume using mixed finite element methods. The recovered flux is used to construct a constant free $a$ posteriori error estimator which is proven to be reliable and efficient. Some numerical tests are presented to confirm the theoretical results. Our method works for general order finite volume methods and the recovery-based and residual-based a posteriori error estimators is the first result on a posteriori error estimators for high order finite volume methods.
\end{abstract}

AMS subject classifications: 65N15, 65N30, 65N50

Key words: Finite volume methods, flux recovery, a posteriori error estimates.

\section{Introduction}

In this paper, we consider vertex-centered finite volume methods for solving diffusion type elliptic equation

$$
-\nabla \cdot(K \nabla u)=f \quad \text { in } \Omega,
$$

with suitable Dirichlet or Neumann boundary conditions. Here $\Omega \subset \mathbb{R}^{d}$ is a polyhedral domain $(d \geq 2)$, the diffusion coefficient $K(x)$ is a $d \times d$ symmetric matrix function that is uniformly positive definite on $\Omega$ with components in $L^{\infty}(\Omega)$, and $f \in L^{2}(\Omega)$. An obvious virtue of finite volume method (FVM) is the local conservation property, which can be fundamental for the simulation of many physical models, e.g., in dynamics of of fluids in porous media.

*Corresponding author.

Email: chenlong@math.uci.edu (L. Chen), wangming.pku@gmail.com (M. Wang) 
We shall recover a cell-conservative flux from vertex-centered FVMs and use the recovered flux to construct a constant free a posteriori error estimator, which will be called recovery-based error estimator. We obtain the reliability, i.e., upper bound, of the recovery-based error estimator through the so-called hypercircle method established by Prager and Synge [32]. We establish the efficiency, i.e., the local lower bound, of the recovery-based error estimator by showing that the recovery-based error estimator is locally equivalent to the well-known residual-based a posteriori error estimator. As a by product, we get the reliability of the residual-based error estimator for high order FVMs, which seems difficult to obtain using the same approach as finite element methods (FEMs) due to the loss of the Galerkin orthogonality.

To facilitate the discussion of the results, let us briefly introduce FVM. Many physical models can be written as the following balance equation:

$$
-\int_{\partial b}(K \nabla u) \cdot n d S=\int_{b} f d x \text { for all } b \subset \Omega .
$$

The discretization of (1.2) by choosing an appropriate finite element space $\mathbb{V}$ to approximate $u$ and a finite number of subdomains $b$, the so-called control volume, will be called FVMs. There are mainly two types of FVMs, different in the choice of control volumes. Given a grid $\mathcal{T}$ of $\Omega$, if we choose the cell of $\mathcal{T}$ as the control volume and associate the unknown to cells, we obtain cell-centered FVMs [10,33]; if we construct control volume for each vertex and associate the unknown to vertex, we obtain vertex-centered FVMs $[7,14,21]$. We shall consider vertex-centered FVMs in this paper.

Let $u_{h}$ be a vertex-centered FVM approximation of Eq. (1.1). By solving a local problem with $K \nabla u_{h} \cdot \boldsymbol{n}$ as Neumann boundary condition on each control volume with mixed finite element methods, we are able to recover a cell conservative flux from $u_{h}$. The local problem is well defined since the compatible condition

$$
-\int_{\partial b}\left(K \nabla u_{h}\right) \cdot n d S=\int_{b} f d x,
$$

is built into the vertex-centered FVM. The approximated flux $\sigma_{h}$ will be sought in RaviartThomas (RT) or Brezzi-Douglas-Marini (BDM) spaces, depending on the order of the approximation $u_{h}$. By solving local problems in all control volumes, we obtain a cellconservative flux on the original grid $\mathcal{T}$ and also a refinement of $\mathcal{T}$. Flux recovery techniques based on the solution of FEM have been extensively studied by many researchers; see [11] for $H$ (div)-conforming flux approximation, and $[15,16]$ for cell-conservative flux approximation. Flux recovery techniques based on the solution of linear FVM can be found in [42]. The new proposed flux recovery method based on the solution of FVM seems natural, much simpler and works also for high order FVM methods.

We prove that the recovered cell-conservative flux is of the same order of approximation as the finite volume approximation in the energy norm. Therefore this local recovery procedure provides an efficient way to compute a cell conservative flux for Eq. (1.1). Note that the quadratic finite volume method proposed in [14] on rectangular grids results in 
a symmetric and positive definite algebraic system which is much easier to solve than the indefinite symmetric algebraic system arising from mixed finite element methods. In general, the system from FVMs is non-symmetric but involves less degrees of freedom compared with mixed finite element methods. Attempts to design symmetric finite volume method can be found in [27-31]. Multilevel preconditioner designed for FEMs can be successively applied to vertex-centered FVMs; see the analysis for the traditional FVMs [25] and the numerical results presented in Section 6 for FVMs developed in [14]. Furthermore, each local problem in the recovery process can be solved efficiently and is suitable for parallel computing.

Our main motivation for the recovery of a cell-conservative flux is to provide a constant free a posteriori error estimator for vertex-centered FVMs. Adaptive methods through local mesh refinement are more preferable to locally increase grid densities in the regions of interest, thus saving the computer resources. The a posteriori error estimator is at the heart of such adaptive methods.

However, contrary to FEMs, a posteriori error estimators for FVMs are less developed, and until now only a few results have been obtained in this direction; see $[3,18,24]$ for cell-centered FVMs, and $[1,12,42]$ for vertex-centered FVMs. The main difficulty is the loss of the Galerkin orthogonality. In the existing literature (e.g., [12]), the error estimates for linear FVMs are mostly derived using the special relation between the linear FVMs and linear FEMs and therefore seems difficult to be applied to high order FVMs. More importantly, in the majority of cases, the estimators are not fully computable due to an unknown constant involved in the upper bound.

We propose an a posteriori error estimator based on the hypercircle method. The advantage of the hypercircle method is that there is no unknown constant involved in the upper bound. The constant is simply one. More precisely, let $u_{h}$ be the approximation of a vertex-centered FVM and $\sigma_{h}$ be the cell-conservative flux obtained by solving the local Neumann problem, we shall prove the following a posteriori error estimator:

$$
\left\|\nabla u-\nabla u_{h}\right\| \leq\left\|\sigma_{h}-\nabla u_{h}\right\|
$$

We emphasize that the upper bound (1.3) does not depend on a hidden constant which is not known explicitly or difficult to estimate sharply. The constant free a posteriori error estimator can be safely used as a stopping criteria in the adaptive computation procedure, which could guarantee the error less than a certain tolerance. Here we present the result for the simplest case: $\boldsymbol{K}=\boldsymbol{I}$ and $f$ is piecewise polynomial. For general coefficients and data, additional but high order terms are involved and a generic constant in front of the higher order term is allowed.

We note that a posteriori error estimate based on the hypercircle method has been applied to FEMs recently, including conforming FEMs [9,38], non-conforming FEMs [6], mixed FEMs [4], and discontinuous Galerkin methods [19]. The application of the hypercircle method to FVMs seems new to the best knowledge of the authors. We also note that Bank and Weiser [7] firstly present some a posteriori error estimators for FEMs from 
the view point of solving a local Neumann problem, where some care should be taken to insure the Neumann problem to be well posed; see also [5].

Besides the reliability, we establish the efficiency of the recovery-based error estimator by showing that it is bounded by the residual-based error estimator. More precisely, for a control volume $b$ with regular decomposition, the following inequality holds,

$$
\left\|\sigma_{h}-\nabla u_{h}\right\|_{b}^{2} \leq C\left(h_{b}^{2}\left\|\bar{f}+\Delta u_{h}\right\|_{0, b}^{2}+\sum_{e \in \mathcal{E}(b)} h_{e}\left\|\left[\nabla u_{h} \cdot \boldsymbol{n}\right]\right\|_{0, e}^{2}\right)
$$

where $\bar{f}$ is the $L^{2}$ projection of $f$ on to piecewise polynomial space with appropriate degree, and $\mathcal{E}(b)$ contains all the interior edges of $b$. Therefore the efficiency of the residualbased error estimator implies that of the recovery-based error estimator, and vice versa, the reliability of the recovery-based error estimator implies that of the residual-based error estimator; see the discussion in Section 5 for detail.

Throughout this paper, we shall make use of the standard Sobolev spaces $H^{m}(\Omega)$ provided with the norm $\|\cdot\|_{m, \Omega}$ and seminorm $|\cdot|_{m, \Omega}$. We denote by $\langle\cdot, \cdot\rangle$ the duality pairing between $H^{-s}(\Omega)$ and $H_{0}^{s}(\Omega)$, and $\|\cdot\|_{-s}$ the norm on $H^{-s}(\Omega)$, for $s>0$. We use $c$ and $C$ to denote generic positive constant independent of the variables that appear in the inequalities and especially the grid parameters.

The rest of this paper is organized as follows. In Section 2 we review some existing vertex-centered FVMs. We describe the detailed recovery procedure in Section 3. In Section 4, we give a priori analysis of the recovered flux. In Section 5, we introduce the recovery-based a posteriori error estimator, and establish the reliability and efficiency of the recovery-based a posteriori error estimator as well as the reliability of the residualbased error estimator for vertex-centered FVMs. Finally, we present numerical results to verify our analysis in Section 6.

\section{Vertex-centered finite volume methods}

In this section, we review several vertex-centered FVMs. For the sake of simplicity, we restrict ourselves to two dimensions and homogeneous Dirichlet boundary condition.

Finite volume methods are discretization of the balance equation (1.2) consisting of three approximations:

1. approximate the function $u$ by $u_{h}$ in a $N$-dimensional sub-space $\mathbb{V}$;

2. approximate "arbitrary domain $b \subset \Omega$ " by a finite set of control volumes $\mathcal{B}=\left\{b_{i}, i=\right.$ $1,2, \cdots, M\}$

3. approximate boundary flux $(\boldsymbol{K} \nabla u) \cdot \boldsymbol{n}$ on $\partial b_{i}$ by a discrete one $\left(\boldsymbol{K} \nabla_{h} u_{h}\right) \cdot \boldsymbol{n}$.

We then end up with a method: find $u_{h} \in \mathbb{V}$ such that:

$$
-\int_{\partial b_{i}}\left(K(\boldsymbol{x}) \nabla_{h} u_{h}\right) \cdot \boldsymbol{n} d S=\int_{b_{i}} f d \boldsymbol{x} \text { for all } b_{i} \subset \Omega, i=1,2, \cdots, M .
$$


We call any method in the form (2.1) finite volume methods.

The choice of $\mathbb{V}$ often relates to a triangulation. Let $\mathcal{T}$ be a conforming and shaperegular simplicial triangulation of $\Omega$. With respect to $\mathcal{T}$, we define the well-known $k$ thorder Lagrange finite element space $\mathbb{V}_{\mathcal{T}}^{k}$ in which the function $u$ is approximated

$$
\mathbb{V}_{\mathcal{T}}^{k}=\left\{v \in H_{0}^{1}(\Omega):\left.v\right|_{\tau} \in \mathcal{P}^{k} \text { for all } \tau \in \mathcal{T}\right\},
$$

where $\mathcal{P}^{k}$ is the polynomial space of degree $k$.

The set of control volumes $\mathcal{B}$ will form another partition of approximated domain of $\Omega$. In what follows $\mathcal{T}$ will be called the primary grid, and $\mathcal{B}$ a dual grid of $\mathcal{T}$. The element $b$ of $\mathcal{B}$ is often chosen to be a polygon for practical computation. One popular construction of a linear dual partition $\mathcal{B}$ is given as follows: for each triangle $\tau \in \mathcal{T}$, select a point $C_{\tau} \in \tau$, and connect $C_{\tau}$ to three middle points on the edges of $\tau$, which will divide each triangle in $\mathcal{T}$ into three regions. Let $\mathcal{N}_{\mathcal{T}}$ be the set of interior vertices of grid $\mathcal{T}$. For each vertex $P \in \mathcal{N}_{\mathcal{T}}$, we collect all regions containing this vertex and define it as $b_{P}$. In Fig. 1 , we draw the control volumes for interior vertices.

There are three common choices of $C_{\tau}$ :

- Type $A: C_{\tau}$ is the barycenter of $\tau$.

- Type $B: C_{\tau}$ is the middle point of the longest edge.

- Type $C: C_{\tau}$ is the circumcenter of $\tau$.

Type $A$ is preferable for triangulations composed by equilateral triangles. Type $B$ is better for right triangles, and is convenient for bisection grids. Type $C$ is suitable for Delaunay triangulations. In this case, the edges of the control volumes will be orthogonal to the intersected edges of triangles, and if the grid $\mathcal{T}$ is a Delaunay triangulation, $\mathcal{B}$ will be a Voronoi diagram.

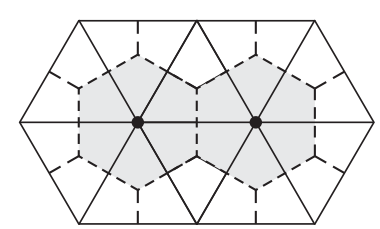

(a) Type $A$

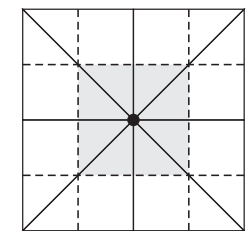

(b) Type $B$

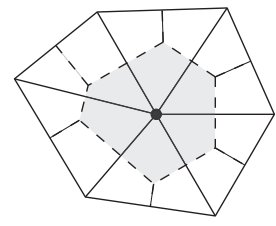

(c) Type $C$

Figure 1: Three types of grids and dual grids. The gray areas are the control volumes of interior nodes. Type $A$ : The points $C_{\tau}$ is the barycenter of $\tau$. Type $B$ : The points $C_{\tau}$ is the middle point of the longest edge. Type $C$ : The points $C_{\tau}$ is the circumcenter of $\tau$.

We now give some examples of vertex-centered FVMs.

Example 2.1. Linear finite volume methods.

We take $\mathbb{V}=\mathbb{V}_{\mathcal{T}}^{1}$ and $\mathcal{B}$ as a linear dual partition. All three types of linear dual partitions can be used for linear finite volume methods. 
Example 2.2. Traditional high order finite volume methods.

We take $\mathbb{V}=\mathbb{V}_{\mathcal{T}}^{k}$. The traditional way to construct a dual partition $\mathcal{B}$ for higher order FVMs is to introduce a control volume for each basis of $\mathbb{V}$, see $[21,22,41]$. For example, for quadratic finite element space $\mathbb{V}_{\mathcal{T}}^{2}$, in addition to the control volumes of vertices, we need control volumes for middle points of edges of $\mathcal{T}$; see (a) and (b) in Fig. 2 for the structure of control volumes. We refer to $\mathrm{Xu}$ and $\mathrm{Zou}$ [41] for the detailed construction of such control volumes and convergence analysis for quadratic FVMs.

A convenient way to construct control volumes for this kind of high order FVMs is to subdivide each triangle into subelements (see Fig. 2(c)), which have the same nodal structure as linear elements. Hence Type $A$ control volumes can then be applied for each subelements; see Vogel, $\mathrm{Xu}$, and Wittum [40].

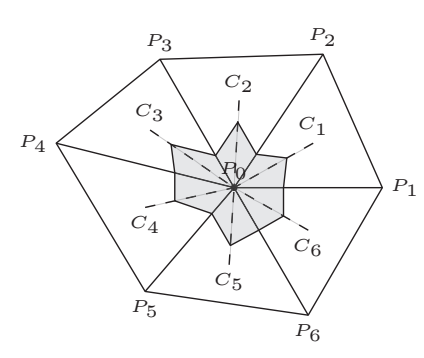

(a) Control volume for $\mathbb{V}_{\mathcal{T}}^{2}$ surrounding vertex $P_{0}$

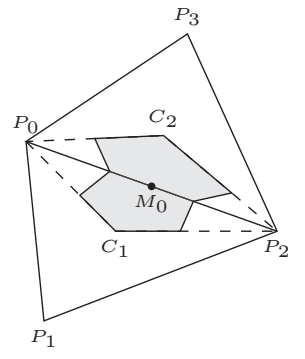

(b) Control volume for $\mathbb{V}_{\mathcal{T}}^{2}$ surrounding middle point of edge $M_{0}$

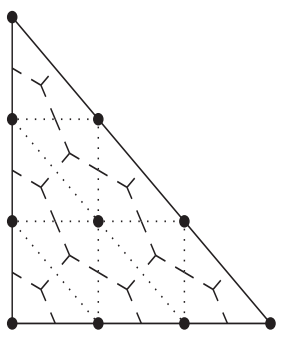

(c) Control volumes (dashed lines) for $\mathbb{V}_{\mathcal{T}}^{3}$

Figure 2: Control volumes for $\mathbb{V}_{\mathcal{T}}^{k}(k=2,3)$. Control volumes associated with vertexes and midpoints of edges for $\mathbb{V}_{\mathcal{T}}^{2}$ in (a) and (b), respectively. Nodes(dots), subelements (dotted lines) and control volumes (dashed lines) for $\mathbb{V}_{\mathcal{T}}^{3}$ in (c).

Example 2.3. High order finite volume methods introduced in Chen [14].

We take $\mathbb{V}=\mathbb{V}_{\mathcal{T}}^{k}$ and still use a linear dual partition $\mathcal{B}$ (only the control volume of vertices are involved). The new class of vertex-centered high order FVMs proposed in [14] can be thought as a hybridization of high order finite element methods and a linear finite volume method. It is obvious that the number of control volumes is less than the dimension of $\mathbb{V}$ in which $u$ is approximated, when $k \geq 2$. Therefore, more equations from FEMs discretization by a hierarchical decomposition of test function spaces are combined to get a closed system; see [14].

Example 2.4. Finite volume methods on rectangular grids.

Let $\mathcal{P}^{m, n}$ denote the polynomials in two variables $x, y$, the maximum degrees of which are respectively $m$ in $x$, and $n$ in $y$. For a rectangular grid $\mathcal{T}$, the $k$-th finite element spaces associated with $\mathcal{T}$ are defined by

$$
\mathbb{V}_{\mathcal{T}}^{k}=\left\{v \in H_{0}^{1}(\Omega):\left.v\right|_{Q} \in \mathcal{P}^{k, k} \text { for all } Q \in \mathcal{T}\right\} .
$$




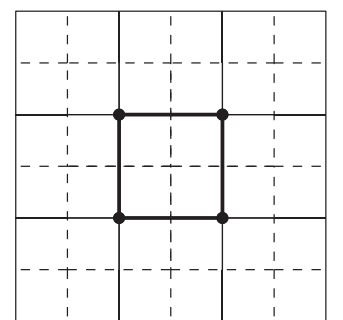

(a) Control volumes for bilinear finite element space $V_{\mathcal{T}}^{1}$

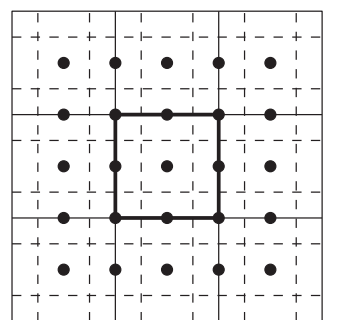

(b) Control volumes for biquadratic finite element space $V_{\mathcal{T}}^{2}$

Figure 3: Control volumes for bilinear FVM and biquadratic FVM. Nodes(dots), elements (thick solid lines), and control volumes (dashed lines).

Traditional FVMs on rectangular grid $\mathcal{T}$ takes $\mathbb{V}$ as $\mathbb{V}_{\mathcal{T}}^{k}$; see [21,26,35]. Control volumes are chosen as rectangles surrounding each degree of freedom (vertices, edge centers, ect.); see Fig. 3 of the control volumes for bilinear and biquadratic FVMs, respectively.

Another kind of higher order FVM takes $\mathbb{V}$ as $\mathbb{V}_{\mathcal{T}}^{k}$ of $H_{0}^{1}(\Omega)$, and employs the simple dual partition control volumes (a) as shown in Fig. 3. Again, more equations from FEM discretization are combined to form a closed system; see [14] for detail.

In the following section, we will focus on FVMs introduced in Example 2.3 to illustrate our ideas. It is straightforward to extend our algorithm and theory to other cases.

\section{Local reconstruction of a cell conservative flux}

In this section, we will give a local reconstruction of a cell conservative flux based on vertex-centered FVMs. We denote by $u_{h} \in \mathbb{V}_{\mathcal{T}}^{k}$ the solution of a $k$-th order vertex-centered FVM for solving (1.1).

Consider the following local Neumann problem on each control volume $b \in \mathcal{B}$,

$$
\begin{cases}-\nabla \cdot(K \nabla u)=f & \text { in } b, \\ K \nabla u \cdot \boldsymbol{n}=\boldsymbol{K} \nabla u_{h} \cdot \boldsymbol{n} & \text { on } \partial b .\end{cases}
$$

The local problem (3.1) is well defined due to the following orthogonality result

$$
\left\langle\boldsymbol{K} \nabla\left(u-u_{h}\right) \cdot \boldsymbol{n}, 1\right\rangle_{\partial b}=\int_{\partial b} \boldsymbol{K} \nabla\left(u-u_{h}\right) \cdot \boldsymbol{n d} s=0 \quad \text { for } b \in \mathcal{B} .
$$

For any $\omega \subset \Omega$, we introduce the space $H($ div; $\omega)$ as

$$
H(\operatorname{div} ; \omega)=\left\{\boldsymbol{\tau} \in\left(L^{2}(\omega)\right)^{d}: \operatorname{div} \boldsymbol{\tau} \in L^{2}(\omega)\right\} .
$$


For $g \in H^{-\frac{1}{2}}(\partial \omega)$, we introduce the subspace $H_{g}(\operatorname{div} ; \omega)$ as

$$
H_{g}(\operatorname{div} ; \omega)=\left\{\boldsymbol{\tau} \in H(\operatorname{div} ; \omega): \boldsymbol{\tau} \cdot \boldsymbol{n}=g \text { in } H^{-\frac{1}{2}}(\partial \omega)\right\} .
$$

Denote $L_{0}^{2}(\omega)$ as the subspace of all $L^{2}$-functions over $\omega$ having mean value zero,

$$
L_{0}^{2}(\omega)=\left\{v \in L^{2}(\omega): \int_{\omega} v \mathrm{~d} x=0\right\} .
$$

With the choice of $\omega=b$ and $g=K \nabla u_{h} \cdot n$ on $\partial b$, the mixed formulation of the local problem (3.1) reads as: find $\left(\sigma_{b}, u_{b}\right) \in H_{g}(\operatorname{div} ; b) \times L_{0}^{2}(b)$ such that

$$
\begin{cases}\left(\boldsymbol{K}^{-1} \sigma_{b}, \boldsymbol{\tau}\right)+\left(\operatorname{div} \boldsymbol{\tau}, u_{b}\right)=0 & \text { for all } \boldsymbol{\tau} \in H_{0}(\operatorname{div} ; b), \\ \left(\operatorname{div} \sigma_{b}, v\right)=-(f, v) & \text { for all } v \in L_{0}^{2}(b) .\end{cases}
$$

Suppose that $\mathcal{T}_{\omega}$ is a shape regular triangulation of $\omega$, the standard $H($ div; $\omega)$ conforming RT spaces $\mathcal{R} \mathcal{T}_{g}^{k}(\omega)$ (for $k \geq 0$ ) and BDM spaces $\mathcal{B D} \mathcal{M}_{g}^{k}(\omega)$ (for $k \geq 1$ ) are defined by

$$
\begin{aligned}
& \mathcal{R} \mathcal{T}_{g}^{k}(\omega)=\left\{\sigma \in H_{g}(\operatorname{div} ; \omega):\left.\sigma\right|_{\tau} \in\left(\mathcal{P}^{k}\right)^{d}+x \mathcal{P}^{k} \text { for all } \tau \in \mathcal{T}_{\omega}\right\}, \\
& \mathcal{B} \mathcal{D} \mathcal{M}_{g}^{k}(\omega)=\left\{\sigma \in H_{g}(\operatorname{div} ; \omega):\left.\sigma\right|_{\tau} \in\left(\mathcal{P}^{k}\right)^{d} \text { for all } \tau \in \mathcal{T}_{\omega}\right\}
\end{aligned}
$$

For convenience, denote $\mathcal{R} \mathcal{T}_{g}^{k-1}(\omega)$ and $\mathcal{B D} \mathcal{M}_{g}^{k}(\omega)$ by $\Sigma_{g}^{k}(\omega)$ for $k \geq 1$.

We also introduce a finite element space $\mathcal{Q}_{0}^{k}(\omega)$ for $L_{0}^{2}(\omega)$,

$$
\mathcal{Q}_{0}^{k}(\omega)=\left\{v \in L_{0}^{2}(\omega):\left.v\right|_{\tau} \in \mathcal{P}^{k} \text { for all } \tau \in \mathcal{T}_{\omega}\right\}
$$

If $\omega=\Omega$, we may omit the subscript $\Omega$ when there is no danger of ambiguity.

The intersection of the primary grid $\mathcal{T}$ and dual grid $\mathcal{B}$ yields a third grid $\mathcal{T}^{\prime}$. For each control volume $b \in \mathcal{B}$, connecting corners of the control volume to its central vertex (i.e., vertex on the primary grid $\mathcal{T}$ ), yields a triangulation of the control volume; see Fig. 4 .

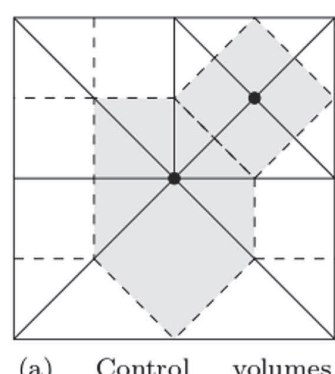

(a) Control volumes (shaded domains)

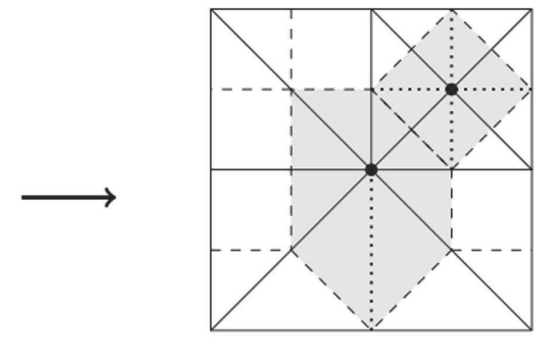

(b) Decomposition of control volumes (dotted lines are drawn)

Figure 4: Construction of the refinement grid $\mathcal{T}^{\prime}$ for type $B$ control volume. 
Notice that for type $B$ dual grid, the third triangulation $\mathcal{T}^{\prime}$, which is a refinement of $\mathcal{T}$, can be obtained via the newest vertex bisection.

The classical mixed finite element method approximation of the variational problem (3.3) reads as: find $\left(\sigma_{h, b}, u_{h, b}\right) \in \Sigma_{g}^{k}(b) \times \mathcal{Q}_{0}^{k-1}(b)$ such that

$$
\begin{cases}\left(\boldsymbol{K}^{-1} \sigma_{h, b}, \boldsymbol{\tau}\right)+\left(\operatorname{div} \boldsymbol{\tau}, u_{h, b}\right)=0 & \text { for all } \boldsymbol{\tau} \in \Sigma_{0}^{k}(b), \\ \left(\operatorname{div} \sigma_{h, b}, v\right)=-(f, v) & \text { for all } v \in \mathcal{Q}_{0}^{k-1}(b) .\end{cases}
$$

We are now in a position to introduce our flux recovery algorithm.

Algorithm 3.1. $\sigma_{h}=$ fluxrecovery $\left(u_{h}\right)$.

Step 1 Solve the local problem (3.5) in each control volume $b \in \mathcal{B}$ to get $\sigma_{h, \mathcal{T}^{\prime}}=\sum_{b \in \mathcal{B}} \sigma_{h, b}$ on the fine grid $\mathcal{T}^{\prime}$.

Step 2 Interpolate the flux $\sigma_{h, \mathcal{T}}$ to the grid $\mathcal{T}$.

Although the construction is carried out over a single control volume at a time, it is ensured that the normal component $\sigma_{h} \cdot \boldsymbol{n}$ is continuous across the element interfaces and that the discrete conservation law holds locally on both the refinement grid $\mathcal{T}^{\prime}$ and the primary grid $\mathcal{T}$. Also, the computation of the local problem is efficient, and is suitable for parallel computing.

\section{A priori analysis of the recovered flux}

In this section, we establish a priori analysis of the recovered flux. We shall prove that the recovered flux is of the same order of approximation as the finite volume approximation in the energy norm.

For a domain $\omega \subset \Omega$, define the $K$-inner product $(\boldsymbol{u}, \boldsymbol{v})_{\boldsymbol{K}, \omega}=\int_{\omega} \boldsymbol{K} \boldsymbol{u v} \mathrm{d} x$, for $\boldsymbol{u}, \boldsymbol{v} \in$ $\left(L^{2}(\omega)\right)^{d}$. The induced norm is denoted by $\|\cdot\|_{K, \omega}$. Define the bilinear form $a(u, v)$ on $H_{0}^{1}(\omega) \times H_{0}^{1}(\omega)$ as

$$
a(u, v)=\int_{\omega}(K(x) \nabla u) \cdot \nabla v \mathrm{~d} x .
$$

It is obvious that $a(u, v)$ is an inner product defined on $H_{0}^{1}(\omega)$. The induced energy norm is denoted by $\|\cdot \mid\|_{\omega}$. Clearly, $a(u, v)=(\nabla u, \nabla v)_{K, \omega}$. The $a$ priori error analysis is given as follows.

Theorem 4.1 (A priori error analysis of recovered flux). Suppose that $u$ is the weak solution of (1.1) and $u \in H^{k+1}(\Omega)(k \geq 1)$. Let $\sigma=K \nabla u$ and $u_{h}$ be the solution of a $k$-th order FVM, and $\sigma_{h}$ the recovered flux given by Algorithm 3.1 using $u_{h}$. We have the following a priori error analysis for the recovered flux $\sigma_{h}$ on each control volume $b \in \mathcal{B}$

$$
\left\|\boldsymbol{K}^{-\frac{1}{2}}\left(\boldsymbol{\sigma}-\boldsymbol{\sigma}_{h}\right)\right\|_{0, b} \leq C(\boldsymbol{K})\left(h^{k}\|u\|_{k+1, b}+\left\|u-u_{h} \mid\right\|_{b}\right),
$$


where $C(\boldsymbol{K})$ depends on the maximal condition number of $\boldsymbol{K}$ over $b$. Further more, if $\left\|u-u_{h}\right\|_{\Omega} \leq$ $h^{k}\|u\|_{k+1, \Omega}$, we have

$$
\left\|\boldsymbol{K}^{-\frac{1}{2}}\left(\boldsymbol{\sigma}-\boldsymbol{\sigma}_{h}\right)\right\|_{0, \Omega} \leq C(\boldsymbol{K}) h^{k}\|u\|_{k+1, \Omega} .
$$

Proof. Suppose that $\tilde{\sigma}_{h, b}$ is the solution of the problem (3.5) using exact Neumann boundary condition on $\partial b$, i.e., $g=\boldsymbol{K} \nabla u \cdot \boldsymbol{n}$ on $\partial b$. Then

$$
\begin{aligned}
\left\|\boldsymbol{K}^{-\frac{1}{2}}\left(\boldsymbol{\sigma}-\boldsymbol{\sigma}_{h}\right)\right\|_{0, b} & \leq\left\|\boldsymbol{K}^{-\frac{1}{2}}\left(\boldsymbol{\sigma}-\tilde{\boldsymbol{\sigma}}_{h, b}\right)\right\|_{0, b}+\left\|\boldsymbol{K}^{-\frac{1}{2}}\left(\tilde{\boldsymbol{\sigma}}_{h, b}-\boldsymbol{\sigma}_{h}\right)\right\|_{0, b} \\
& \leq C \lambda_{\min }^{-1}(\boldsymbol{K})\left(h_{b}^{k}\|u\|_{k+1, b}+\left\|\boldsymbol{K} \nabla\left(u-u_{h}\right) \cdot \boldsymbol{n}\right\|_{-\frac{1}{2}, \partial b}\right) .
\end{aligned}
$$

Here, we have used the stability result for solving Poisson equation with mixed finite element methods in the estimate of the second term. By the definition of the duality pairing between $H^{-\frac{1}{2}}(\partial b)$ and $H^{\frac{1}{2}}(\partial b)$, and the orthogonality result (3.2), we have

$$
\begin{aligned}
\left\|\boldsymbol{K} \nabla\left(u-u_{h}\right) \cdot \boldsymbol{n}\right\|_{-\frac{1}{2}, \partial b} & =\sup _{\phi \in H^{\frac{1}{2}}(\partial b)} \frac{\left\langle\boldsymbol{K} \nabla\left(u-u_{h}\right) \cdot \boldsymbol{n}, \phi\right\rangle_{\partial b}}{\|\phi\|_{\frac{1}{2}, \partial b}} \\
& =\sup _{\phi \in H^{\frac{1}{2}}(\partial b)} \frac{\left\langle\boldsymbol{K} \nabla\left(u-u_{h}\right) \cdot \boldsymbol{n}, \phi-c\right\rangle_{\partial b}}{\|\phi\|_{\frac{1}{2}, \partial b}} \\
\leq & C(\boldsymbol{K}) h_{b}^{\frac{1}{2}}\left\|\nabla\left(u-u_{h}\right)\right\|_{0, \partial b .}
\end{aligned}
$$

In the last step, we have used

$$
\|\phi-c\|_{\partial b} \leq C h_{b}^{\frac{1}{2}}\|\phi\|_{\frac{1}{2}, \partial b} \quad \text { and } \quad|\boldsymbol{K} \cdot \boldsymbol{n}| \leq \lambda_{\max }(\boldsymbol{K}) .
$$

Denote by $u_{I}$ the $k$-th nodal interpolation of $u$. We have

$$
\begin{aligned}
\left\|\boldsymbol{K} \nabla\left(u-u_{h}\right) \cdot \boldsymbol{n}\right\|_{-\frac{1}{2}, \partial b} & \leq C(\boldsymbol{K}) h_{b}^{\frac{1}{2}}\left\|\nabla\left(u-u_{h}\right)\right\|_{0, \partial b} \\
& \leq C(\boldsymbol{K})\left(\left\|u-u_{h}\right\|_{b}+h_{b} \sum_{\tau \in b}\left\|D^{2} u-D^{2} u_{h}\right\|_{\tau}\right) \\
& \leq C(\boldsymbol{K})\left(\left\|u-u_{h}\right\|_{b}+h_{b} \sum_{\tau \in b}\left(\left\|D^{2} u-D^{2} u_{I}\right\|_{\tau}+\left\|D^{2} u_{I}-D^{2} u_{h}\right\|_{\tau}\right)\right) \\
& \leq C(\boldsymbol{K})\left(\left\|u-u_{h}\right\|_{b}+h_{b}^{k}\|u\|_{k+1, b}+\left\|u_{I}-u_{h}\right\|_{b}\right) \\
& \leq C(K)\left(\left\|u-u_{h}\right\|_{b}+h_{b}^{k}\|u\|_{k+1, b}\right) .
\end{aligned}
$$

In the second inequality, we have used the following scaled trace inequality, which can be easily proved by the standard scaling argument

$$
\|v\|_{0, \partial \tau} \leq C\left(h_{\tau}^{-\frac{1}{2}}\|v\|_{0, \tau}+h_{\tau}^{\frac{1}{2}}\|\nabla v\|_{0, \tau}\right) \quad \text { for all } v \in H^{1}(\tau) .
$$

Therefore, the error bound in (4.2) holds.

The optimal order error estimate of linear and quadratic FVMs, i.e., $\left\|u-u_{h}\right\|_{\Omega} \leq$ $C h^{k}\|u\|_{k+1, \Omega}$ for $k=1,2$, can be found in $[14,41]$. 


\section{A posteriori error estimator based on the hypercircle method}

This section is devoted to the a posteriori error estimate for FVMs. Throughout this section, $u \in H_{0}^{1}(\Omega)$ denotes the weak solution of (1.1) with homogeneous Dirichlet boundary condition, $u_{h} \in \mathbb{V}_{\mathcal{T}}^{k}$ denotes the solution of a $k$-th order FVM, and $\sigma_{h}$ denotes the recovered cell conservative flux from Algorithm 3.1 using $u_{h}$. We introduce the recovery-based a posteriori error estimator associated with $u_{h}$

$$
\eta_{\mathrm{rec}, \tau}=\left\|\boldsymbol{K}^{-1} \sigma_{h}-\nabla u_{h}\right\|_{\boldsymbol{K}, \tau}=\left(\int_{\tau}\left(\sigma_{h}-\boldsymbol{K} \nabla u_{h}\right)\left(\boldsymbol{K}^{-1} \sigma_{h}-\nabla u_{h}\right) \mathrm{d} x\right)^{\frac{1}{2}}
$$

and

$$
\eta_{\mathrm{rec}}=\left(\sum_{\tau \in \mathcal{T}} \eta_{\mathrm{rec}, \tau}^{2}\right)^{\frac{1}{2}}=\left\|\boldsymbol{K}^{-1} \sigma_{h}-\nabla u_{h}\right\|_{\boldsymbol{K}}
$$

We will first show the reliability of the recovery-based error estimator $\eta_{\text {rec, }}$, and then establish the efficiency of the error estimator $\eta_{\text {rec }}$ by showing the equivalence between the recovery-based error estimator and the residual-based error estimator.

\subsection{Reliability of the recovery-based error estimator}

The hypercircle method developed by Prager and Synge [32], see also [36], is based on the orthogonality of the subspace grad $H_{0}^{1}(\Omega)$ and $\operatorname{ker}(\operatorname{div})$ in $\left(L^{2}(\Omega)\right)^{d}$ in the $K$-inner product. For a given $f \in L^{2}(\Omega)$, we define the following affine subspace of $H(\operatorname{div} ; \Omega)$

$$
H_{f}(\operatorname{div} ; \Omega)=\{\sigma \in H(\operatorname{div} ; \Omega): \operatorname{div} \sigma+f=0\} .
$$

Theorem 5.1 (Prager and Synge). Suppose that $u \in H_{0}^{1}(\Omega)$ satisfying $-\operatorname{div}(K \nabla u)=f$. We have the following identity: for any $\sigma \in H_{f}(\operatorname{div} ; \Omega)$ and $v \in H_{0}^{1}(\Omega)$,

$$
\|\nabla u-\nabla v\|_{\boldsymbol{K}}^{2}+\left\|\nabla u-K^{-1} \sigma\right\|_{K}^{2}=\left\|\nabla v-K^{-1} \sigma\right\|_{K}^{2} .
$$

Proof. The proof of (5.2) is a simple consequence of the orthogonality

$$
\left(\nabla u-\nabla v, \nabla u-K^{-1} \sigma\right)_{K}=(\nabla(u-v), K \nabla u-\sigma)=(u-v,-\nabla \cdot(K \nabla u-\sigma))=0 .
$$

The theorem is proved.

The name "hypercircle method" comes from the fact the three functions $\nabla u, \nabla v$ and $K^{-1} \sigma$ lie in a hypercircle in the functions space $\left(L^{2}(\Omega)\right)^{d}$ under the $\boldsymbol{K}$-inner product.

If we choose $v=u_{h} \in \mathbb{V}_{\mathcal{T}}^{k} \subset H_{0}^{1}(\Omega)$ in (5.2), we obtain the inequality

$$
\left\|u-u_{h}\right\| \mid \leq \inf _{\sigma \in H_{f}(\operatorname{div} ; \Omega)}\left\|K^{-1} \sigma-\nabla u_{h}\right\|_{K} .
$$


To get a posteriori error estimator, we can try to find $\sigma_{h}$ in the $H($ div) conforming finite element spaces, when $f$ is a piecewise polynomial function. It can be shown that the approximation attained by the mixed finite element method [8] is optimal. Instead of solving a mixed formulation to obtain an optimal choice, which is computationally expensive, we choose a quasi-optimal one $\sigma_{h}$ constructed from Algorithm 3.1. We then end up with the following reliability of the recovery-based error estimator $\eta_{\text {recc }}$.

Theorem 5.2 (Reliability of the recovery-based error estimator). Let $\bar{f}$ be the $L^{2}$ projection of $f$ on to piecewise polynomial space of degrees at most $k-1$, with respect to the grid $\mathcal{T}$. Let $u$ be the weak solution of Eq. (1.1), $u_{h} \in \mathbb{V}_{\mathcal{T}}^{k}$ be the solution of a $k$-th $F V M$, and $\eta_{\mathrm{rec}}$ be the recovery-based error estimator defined in (5.1) using $u_{h}$. Then the following a priori error bound holds:

$$
\left\|u-u_{h} \mid\right\| \leq \eta_{\text {rec }}+C(K)\|h(f-\bar{f})\| .
$$

Proof. Let $\bar{u} \in H_{0}^{1}(\Omega)$ satisfying

$$
-\operatorname{div}(K \nabla \bar{u})=\bar{f}
$$

Combination of Theorem 5.1 and inequality (5.3) gives us

$$
\left\|\bar{u}-u_{h}\right\| \leq \inf _{\sigma \in H_{\bar{f}}(\operatorname{div} ; \Omega)}\left\|\boldsymbol{K}^{-1} \sigma-\nabla u_{h}\right\|_{\boldsymbol{K}}
$$

The construction of the cell conservative flux $\sigma_{h}$ from Algorithm 3.1 implies that $\operatorname{div} \sigma_{h}=\bar{f}$, which yields

$$
\left\|\bar{u}-u_{h} \mid\right\| \leq \eta_{\text {rec }}
$$

from (5.6) and the definition (5.1) of $\eta_{\text {rec }}$.

The difference of the solutions of (1.1) and (5.5) in the energy norm, $\|u-\bar{u}\| \|$, shall be bounded by

$$
C(\boldsymbol{K})\|h(f-\bar{f})\| \text {. }
$$

The basic triangle inequality concludes the estimate (5.4).

Note that the term (5.7) is known as data oscillation and usually is a high order term $[8,23]$, thus an unknown constant is allowed.

\subsection{Equivalence of the residual-based and recovery-based error estimator}

We prove the efficiency of the recovery-based a posteriori error estimator by establishing the equivalence between the recovery-based error estimator and the residual-based error estimator on each control volume, following Braess and Schöberl [9]. 
Let $\mathcal{E}_{\mathcal{T}^{\prime}}$ denote the set of interior edges of the fine grid $\mathcal{T}^{\prime}$. For $v_{h} \in \mathbb{V}_{\mathcal{T}^{\prime}}^{k}$, the element residual $R_{\tau^{\prime}}\left(v_{h}\right)$ and the jump residual $J_{e^{\prime}}\left(v_{h}\right)$ are defined by

$$
\begin{array}{ll}
R_{\tau^{\prime}}\left(v_{h}\right):=f+\nabla \cdot\left(\boldsymbol{K} \nabla v_{h}\right), & \text { in } \tau^{\prime} \in \mathcal{T}^{\prime}, \\
J_{e^{\prime}}\left(v_{h}\right):=-\left.\boldsymbol{K} \nabla v_{h}\right|_{\tau_{1}^{\prime}} \cdot \boldsymbol{n}_{1, e^{\prime}}-\left.\boldsymbol{K} \nabla v_{h}\right|_{\tau_{2}^{\prime}} \cdot \boldsymbol{n}_{2, e^{\prime}}=\left[\boldsymbol{K} \nabla v_{h} \cdot \boldsymbol{n}_{e^{\prime}}\right]_{e^{\prime}}, & \text { on } e^{\prime} \in \mathcal{E}_{\mathcal{T}^{\prime}},
\end{array}
$$

where $e^{\prime}$ is the common side of elements $\tau_{1}^{\prime}$ and $\tau_{2}^{\prime}$ with unit outward normals $\boldsymbol{n}_{1, e^{\prime}}$ and $n_{2, e^{\prime}}$, respectively, and $\boldsymbol{n}_{e^{\prime}}=\boldsymbol{n}_{1, e^{\prime}}$. The residual-based error estimator associated with $v_{h}$ is defined as

$$
\eta_{\mathrm{R}, \tau^{\prime}}\left(v_{h}\right)=\left(h_{\tau^{\prime}}^{2}\left\|\bar{R}_{\tau^{\prime}}\left(v_{h}\right)\right\|_{0, \tau^{\prime}}^{2}+\sum_{e^{\prime} \in \partial \tau^{\prime}} h_{e^{\prime}}\left\|J_{e^{\prime}}\left(v_{h}\right)\right\|_{0, e^{\prime}}^{2}\right)^{\frac{1}{2}} \text { for } \tau^{\prime} \in \mathcal{T}^{\prime},
$$

where $\bar{R}_{\tau^{\prime}}\left(v_{h}\right) \in \mathcal{P}_{k-1}\left(\tau^{\prime}\right)$ is the $L^{2}$-projection of $R_{\tau^{\prime}}\left(v_{h}\right), h_{\tau^{\prime}}=\left|\tau^{\prime}\right|^{\frac{1}{2}}$, and $h_{e^{\prime}}=\left(h_{\tau_{1}^{\prime}}+h_{\tau_{2}^{\prime}}\right) / 2$. We will skip the notation $v_{h}$ in $\eta_{\mathrm{R}, \tau^{\prime}}\left(v_{h}\right)$ if there is no ambiguity.

For a subset $\mathcal{M}^{\prime} \subset \mathcal{T}^{\prime}$, we define

$$
\eta_{\mathrm{R}, \mathcal{M}^{\prime}}=\left(\sum_{\tau^{\prime} \in \mathcal{M}^{\prime}} \eta_{\mathrm{R}, \tau^{\prime}}^{2}\right)^{\frac{1}{2}} .
$$

Assume that $\sigma_{h}$ is the recovered flux from Algorithm 3.1 using $u_{h}$. The global flux correction can be defined as

$$
\sigma^{\Delta}=\sigma_{h}-K \nabla u_{h}
$$

With the definition of the element residual $R_{\tau^{\prime}}\left(u_{h}\right)$ and the jump residual $J_{e^{\prime}}\left(u_{h}\right)$, the restriction of $\sigma^{\Delta}$ to the control volume $b \in \mathcal{B}$ is characterized by the following equations

$$
\begin{cases}\operatorname{div} \sigma^{\Delta}=-\bar{R}_{\tau^{\prime}}\left(u_{h}\right) & \text { for } \tau^{\prime} \in b, \\ {\left[\sigma^{\Delta} \cdot n_{e^{\prime}}\right]_{e^{\prime}}=-J_{e^{\prime}}\left(u_{h}\right)} & \text { for } e^{\prime} \in \mathcal{E}(b), \\ \boldsymbol{\sigma}^{\Delta} \cdot \boldsymbol{n}_{e^{\prime}}=0 & \text { for } e^{\prime} \in \partial b .\end{cases}
$$

Since $u_{h}$ is chosen from the finite element space $\mathbb{V}_{\mathcal{T}}^{k}$, and $J_{\mathcal{e}^{\prime}}\left(u_{h}\right)$ is nonzero except for edges lie on the primary grid (solid edges inside each control volume, see Fig. 4), the global flux correction $\sigma^{\Delta}$ does not belong to the continuous $H$ (div) conforming spaces. We have to proceed in the discontinuous spaces. To this end, for a control volume $b \in \mathcal{B}$, we introduce the broken spaces $\mathcal{R} \mathcal{T}_{-1}^{k}(b)$ and broken spaces $\mathcal{B D}_{\mathcal{D}}^{k}{ }_{-1}(b)$ as

$$
\begin{aligned}
& \mathcal{R} \mathcal{T}_{-1}^{k}(b):=\left\{\sigma \in\left[L^{2}(b)\right]^{d}:\left.\sigma\right|_{\tau^{\prime}} \in\left(\mathcal{P}^{k}\left(\tau^{\prime}\right)\right)^{d}+x \mathcal{P}^{k}\left(\tau^{\prime}\right) \text { for all } \tau^{\prime} \in b\right\}, \\
& \mathcal{B D} \mathcal{M}_{-1}^{k}(b):=\left\{\sigma \in\left[L^{2}(b)\right]^{d}:\left.\sigma\right|_{\tau^{\prime}} \in\left(\mathcal{P}^{k}\left(\tau^{\prime}\right)\right)^{d} \text { for all } \tau^{\prime} \in b\right\} .
\end{aligned}
$$

Again, for convenience, denote $\mathcal{R} \mathcal{T}_{-1}^{k-1}(b)$ and $\mathcal{B D} \mathcal{M}_{-1}^{k}(b)$ as $\Sigma_{-1}^{k}(b)$ for $k \geq 1$. We will also introduce the subspace of the broken spaces $\Sigma_{-1}^{k}(b)$ as

$$
\Sigma_{-1,0}^{k}(b)=\left\{\boldsymbol{\sigma} \in \Sigma_{-1,0}^{k}(b): \sigma \cdot \boldsymbol{n}=0 \text { on } \partial b\right\} .
$$


The broken spaces are equipped with the norm

$$
\|\sigma\|_{K^{-1}, b}=\sqrt{(\sigma, \sigma)_{K^{-1}, b^{\prime}}}
$$

where the $\boldsymbol{K}^{-1}$ inner product on $\left(L^{2}(b)\right)^{d}$ is defined as

$$
(\boldsymbol{u}, \boldsymbol{v})_{\boldsymbol{K}^{-1}, b}=\left(\boldsymbol{K}^{-1} \boldsymbol{u}, \boldsymbol{v}\right) \text { for all } \boldsymbol{u}, \boldsymbol{v} \in\left(L^{2}(b)\right)^{d} .
$$

Obviously, the restriction of $\sigma^{\Delta}$ on $b$ belongs to the space $\Sigma_{-1,0}^{k}(b)$, and

$$
\left\|\sigma^{\Delta}\right\|_{K^{-1}, b}=\eta_{\text {rec }, b}:=\left\|\sigma_{h}-\nabla u_{h}\right\|_{K^{-1}, b} .
$$

For $\sigma \in \Sigma_{-1,0}^{k}(b)$, we have $\operatorname{div} \sigma \in H^{-1}(b)$ and

$$
\langle\operatorname{div} \sigma, v\rangle=\sum_{\tau^{\prime} \in b} \int_{\tau^{\prime}} \operatorname{div} \sigma v+\sum_{e^{\prime} \in \mathcal{E}_{b}} \int_{e^{\prime}}\left[\boldsymbol{\sigma} \cdot \boldsymbol{n}_{e^{\prime}}\right] v \quad \text { for all } v \in H_{0}^{1}(b) .
$$

The representation of $\operatorname{div} \sigma$ in the space $H^{-1}(b)$ inspires us to define the grid dependent seminorm on $\Sigma_{-1,0}^{k}(b)$

$$
\|\operatorname{div} \sigma\|_{-1, h, b}=\left(\sum_{\tau^{\prime} \in b} h_{\tau^{\prime}}^{2}\|\operatorname{div} \sigma\|_{0, \tau^{\prime}}^{2}+\sum_{e^{\prime} \in \mathcal{E}_{b}} h_{e^{\prime}}\left\|\left[\sigma \cdot n_{e^{\prime}}\right]\right\|_{0, e^{\prime}}^{2}\right)^{\frac{1}{2}} .
$$

It is easy to verify that $\|\operatorname{div}(\cdot)\|_{-1, h, b}$ defines a norm on $\Sigma_{-1,0}^{k}(b) / \operatorname{ker}(\operatorname{div})$.

The following norm equivalence on the quotient space $\Sigma_{-1,0}^{k}(b) / \operatorname{ker}(\operatorname{div})$ is evident.

Lemma 5.1 (Norm equivalence). For any $b \in \mathcal{B}$, we have the following norm equivalence on the quotient space $\Sigma_{-1,0}^{k}(b) / \operatorname{ker}(\operatorname{div})$, for all $\sigma \in \Sigma_{-1,0}^{k}(b) / \operatorname{ker}(\operatorname{div})$,

$$
c(\boldsymbol{K})\|\boldsymbol{\sigma}\|_{\boldsymbol{K}^{-1}, b} \leq\|\operatorname{div} \boldsymbol{\sigma}\|_{-1, h, b} \leq C(\boldsymbol{K})\|\boldsymbol{\sigma}\|_{\boldsymbol{K}^{-1}, b} .
$$

Consequently, the global flux correction $\sigma^{\Delta}$ defined in (5.10) satisfies

$$
c(\boldsymbol{K})\left\|\sigma^{\Delta}\right\|_{\boldsymbol{K}^{-1}, b} \leq\left\|\operatorname{div} \boldsymbol{\sigma}^{\Delta}\right\|_{-1, h, b} \leq C(\boldsymbol{K})\left\|\boldsymbol{\sigma}^{\Delta}\right\|_{\boldsymbol{K}^{-1}, b} .
$$

Proof. Note that both $\|\cdot\|_{\boldsymbol{K}^{-1}, b}$ and $\|\operatorname{div}(\cdot)\|_{-1, h, b}$ define norms on the finite dimensional quotient space $\Sigma_{-1,0}^{k}(b) / \operatorname{ker}(\operatorname{div})$, the norm equivalence (5.18) holds obviously.

To verify (5.19), it suffices to show that $\sigma^{\Delta}$ is orthogonal to the kernel space $\Sigma_{-1,0}^{k}(b) \cap$ $\operatorname{ker}(\operatorname{div})$, with respect to $(\cdot, \cdot)_{K^{-1}}$ inner product. It is easy to check that

$$
\Sigma_{-1,0}^{k}(b) \cap \operatorname{ker}(\operatorname{div})=\Sigma_{0}^{k}(b) \cap \operatorname{ker}(\operatorname{div}) .
$$

Therefore we only need to prove that $\sigma^{\Delta}$ is orthogonal to the space $\Sigma_{0}^{k}(b) \cap \operatorname{ker}(\operatorname{div})$.

For $\tau \in \Sigma_{0}^{k}(b) \cap \operatorname{ker}(\operatorname{div})$, from (3.5), the construction of $\sigma_{h}$, we have

$$
\left(\boldsymbol{\sigma}^{\Delta}, \boldsymbol{\tau}\right)_{\boldsymbol{K}^{-1}, b}=\left(\boldsymbol{K}^{-1} \boldsymbol{\sigma}_{h}-\nabla u_{h}, \boldsymbol{\tau}\right)_{b}=-\left(u_{h, b}, \nabla \cdot \boldsymbol{\tau}\right)_{b}+\left(u_{h}, \nabla \cdot \boldsymbol{\tau}\right)_{b}=0 .
$$

Here recall that $u_{h, b} \in \mathcal{Q}_{g}^{k-1}(b)\left(\right.$ with $\left.g=K \nabla u_{h} \cdot \boldsymbol{n}\right)$ is the piecewise polynomial function on $b$ given by the mixed formulation (3.5). This ends the proof of (5.19). 
Notice that $\left\|\sigma^{\Delta}\right\|_{K^{-1}, b}=\eta_{\mathrm{rec}, b}$ and $\left\|\operatorname{div} \sigma^{\Delta}\right\|_{-1, h, b}=\eta_{\mathrm{R}, b}$, we are ready to present our main theorem in this subsection.

Theorem 5.3 (Local equivalence of recovery-based and residual-based error estimator). For $b \in \mathcal{B}$, let the recovery-based error estimator $\eta_{\mathrm{rec}, b}$ and the residual-based error estimator $\eta_{\mathrm{R}, b}$ be defined in (5.15) and (5.9), respectively, we therefore have

$$
c(\boldsymbol{K}) \eta_{\mathrm{rec}, b} \leq \eta_{\mathrm{R}, b} \leq C(\boldsymbol{K}) \eta_{\mathrm{rec}, b} .
$$

The efficiency of the residual-based error estimator can be attained through the standard bubble function technique introduced by Verfürth [39]. Note that the lower bound holds for any finite element function $v_{h} \in \mathbb{V}_{k}$ by checking the details of the proof in Verfürth [39].

Lemma 5.2 (Efficiency of the residual-based error estimator $\eta_{R}$ ). Suppose that $u \in H_{0}^{1}(\Omega)$ is the weak solution of Eq. (1.1). We have the following estimate: for $v_{h} \in \mathbb{V}_{k, b}$,

$$
\eta_{\mathrm{R}, b}\left(v_{h}\right) \leq C(\boldsymbol{K})\left(\left\|u-v_{h}\right\|_{b}+\sum_{\tau^{\prime} \in b} h_{\tau^{\prime}}\|f-\bar{f}\|_{0, \tau^{\prime}}\right),
$$

where $\bar{f} \in \mathcal{P}^{k-1}$ is the $L^{2}$ projection of $f$ on $\tau^{\prime}$.

As a consequence of Theorem 5.3 and Lemma 5.2, we obtain the efficiency of the recovery-based error estimator $\eta_{\text {rec }}$.

Corollary 5.1 (Efficiency of the recovery-based error estimator $\eta_{\mathrm{rec}}$ ). Suppose that $u_{h}$ is the solution given by a $k$-th order FVM, and $\eta_{\mathrm{rec}, \tau}$ is the recovery-based error estimator, then we have

$$
\eta_{\mathrm{rec}, \tau} \leq C(\boldsymbol{K})\left(\sum_{b \cap \tau \neq \varnothing}\left\|u-u_{h}\right\|_{b}+\sum_{b \cap \tau \neq \varnothing} \sum_{\tau^{\prime} \in b} h_{\tau^{\prime}}\|f-\bar{f}\|_{0, \tau^{\prime}}\right) .
$$

The following monotonicity of the residual-based error estimator with respect to the grid can be found in [12].

Lemma 5.3 (Monotonicity of the residual-based error estimator). Assume $\mathcal{T}^{\prime}$ is a refinement of $\mathcal{T}$. For $v_{h} \in \mathbb{V}_{\mathcal{T}}^{k}$, we have

$$
\eta_{\mathrm{R}, \mathcal{T}^{\prime}}\left(v_{h}\right) \leq \eta_{\mathrm{R}, \mathcal{T}}\left(v_{h}\right) .
$$

Another consequence of the equivalence (5.20) is the reliability of the residual-based error estimator $\eta_{\mathrm{R}}$. Note that the reliability of residual-based error estimator for high order FVMs seems difficult to get following the same way as FVMs, due to the loss of the Galerkin orthogonality. Combination of Theorem 5.2, Lemma 5.2, Lemma 5.3, and Corollary 5.1 gives us the result without using the Galerkin orthogonality.

Corollary 5.2 (Reliability and efficiency of the residual-based error estimator $\eta_{\mathrm{R}}$ ). Let $u_{h}$ be the solution of a $k$-th order FVMs. The residual-based error estimator $\eta_{\mathrm{R}}$ is both reliable and efficient, i.e., we have

$$
\eta_{\mathrm{R}, \mathcal{T}}-C\|h(f-\bar{f})\| \leq C(\boldsymbol{K})\left\|u-u_{h}\right\| \leq \eta_{\mathrm{R}, \mathcal{T}}+C\|h(f-\bar{f})\| .
$$


Remark 5.1. The significance of (5.23) is that the subroutine of computing standard residual based error estimator for FEMs can be applied for FVMs without additional coding.

\section{Numerical examples}

To confirm the theoretical results established in the previous sections, numerical experiments are carried out for three test examples. The simulation is implemented using the MATLAB software package $i$ FEM [13] and tested on a machine with 2.8GHz Intel Xeon processor.

The optimal order approximation of the recovered flux is tested for the Poisson equation with smooth solutions in Example 6.1. The other two Examples are devoted to test the performance of the flux recovery method when the exact solution is singular. In each example, both linear and quadratic FVMs in [14] are employed for the recovery procedure, and only type $B$ linear dual partition is considered for convenience. For linear FVM, the flux is recovered in $\mathcal{R} \mathcal{T}^{0}$, and for quadratic FVM, the flux is recovered in $\mathcal{B D} \mathcal{M}^{1}$, and the flux recovery procedure will be referred to linear flux recovery and quadratic flux recovery, respectively.

Compared with mixed FEM, the size of the matrix arising from vertex-centered FVM discretization is smaller. More specifically, the size of the matrix arising from linear and quadratic FVMs discretization is about 5 times and 2 times less than associate mixed FEM, respectively. Furthermore, the linear systems obtained from FVMs discretization are much more easier to solve using preconditioned Krylov subspace methods. $\mathcal{V}$-cycle preconditioned Conjugate Gradient (CG) method and $\mathcal{V}$-cycle preconditioned Generalized Minimal Residual (GMRES) method are used to solve the linear system discretized from linear and quadratic FVMs, respectively. Iteration steps and CPU times required are reported for each test example; see Tables 2, 4, 6. In the tables, \#Nodes denotes the number of nodes, and $N=\#$ DOF denotes the number of degrees of freedom. All the results reported from Tables 2, 4, 6 show that multilevel preconditioner designed for FEMs can be successively applied to vertex-centered FVMs.

To reduce error caused by numerical quadratures, we use quadratic and cubic numerical quadratures to compute the right hand side for linear and quadratic FVMs, respectively. The log-log plots for the error are adopted, and polynomial fit is used to calculate the decay order of the error.

Example 6.1. Optimal order approximation of the recovered flux.

In this example, we show the optimal approximation order of the recovered flux. We take $K=I$, the identity matrix and $\Omega=(0,1)^{2}$, i.e., the Poisson equation on the unit square. The exact solution is chosen as

$$
u=\cos (\pi x) \cos (\pi y)-1,
$$

with Dirichlet boundary condition. 
Table 1: Error for flux recovered from linear and quadratic FVMs.

\begin{tabular}{||c|c|c||}
\hline \# Nodes & ErrRT0 & ErrBDM1 \\
\hline 1089 & 0.0629 & $2.2326 e-3$ \\
4225 & 0.0314 & $5.6138 e-4$ \\
16641 & 0.0157 & $1.4074 e-4$ \\
66049 & $7.8696 e-3$ & $3.5241 e-5$ \\
263169 & $3.9348 e-3$ & $8.8834 e-6$ \\
1050625 & $1.9674 e-3$ & $2.7011 e-6$ \\
\hline
\end{tabular}

Table 2: Iteration steps and CPU cost for solving the linear system discretized from linear and quadratic FVMs with $\mathcal{V}$-cycle preconditioned Krylov subspace methods (Example 6.1).

(a) $\mathcal{V}$-cycle preconditioned $\mathrm{CG}$ method for linear FVM equation

\begin{tabular}{||c|c|c|c||}
\hline \# Nodes & \# DOF & iterStep & time (s) \\
\hline 1,089 & 1,089 & 8 & 0.1993 \\
4,225 & 4,225 & 8 & 0.0339 \\
16,641 & 16,641 & 8 & 0.1368 \\
66,049 & 66,049 & 8 & 0.6437 \\
263,169 & 263,169 & 8 & 3.0061 \\
$1,050,625$ & $1,050,625$ & 8 & 14.232 \\
\hline
\end{tabular}

(b) $\mathcal{V}$-cycle preconditioned GMRES method for quadratic FVM equation

\begin{tabular}{||c|c|c|c||}
\hline \# Nodes & \# DOF & iterStep & time (s) \\
\hline 1,089 & 4,225 & 9 & 0.3904 \\
4,225 & 16,641 & 9 & 0.1539 \\
16,641 & 66,049 & 9 & 0.5753 \\
66,049 & 263,169 & 9 & 2.6325 \\
263,169 & $1,050,625$ & 9 & 14.362 \\
$1,050,625$ & $4,198,401$ & 9 & 58.354 \\
\hline
\end{tabular}

We present the numerical result in Table 1 and plot the convergence rate for the linear and quadratic flux recovery in Fig. 5. The approximation errors ErrRT0 $=\left\|\nabla u-\sigma_{\text {rec }}^{R T^{0}}\right\|$ and ErrBDM1 $=\left\|\nabla u-\sigma_{\text {rec }}^{B D M^{1}}\right\|$ measure the difference of the exact flux $\nabla u$ and recovered flux in the $L^{2}$ norm, and again quadratic and cubic numerical quadratures are employed, respectively.

From Table 1 and Fig. 5, it is evident that the recovered flux achieves optimal order

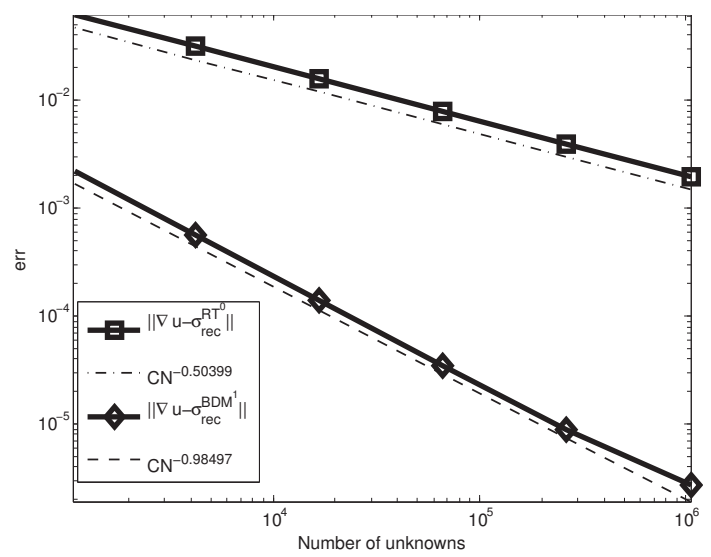

Figure 5: Convergence rate for linear and quadratic flux recovery. 
in the $L^{2}$ norm, i.e., first-order and second-order convergence rate for the flux obtained from the linear and quadratic flux recovery procedure, respectively.

Example 6.2. The a posteriori error estimator: Kellogg problem.

We show the efficiency of the recovery-based a posteriori error estimator with discontinuous coefficient problem. The bulk marking strategy by Dörfler [17] with $\theta=0.2$ is adopted in our simulation for marking. Marked elements are refined by the newest vertex bisection.

We employ a test example designed by Kellogg [20]. Consider the partial differential equation (1.1) with $\Omega=(-1,1)^{2}$ and the coefficient matrix $K$ is piecewise constant: in the first and third quadrants, $K=a_{1} \boldsymbol{I}$; in the second and fourth quadrants, $\boldsymbol{K}=a_{2} \boldsymbol{I}$. For $f=0$, the exact solution in polar coordinates has been chosen to be $u(r, \theta)=r^{\gamma} \mu(\theta)$, where

$$
\mu(\theta)= \begin{cases}\cos \left(\left(\frac{\pi}{2}-\sigma\right) \gamma\right) \cos \left(\left(\theta-\frac{\pi}{2}+\rho\right) \gamma\right), & \text { if } 0 \leq \theta \leq \frac{\pi}{2} \\ \cos (\rho \gamma) \cos ((\theta-\pi+\sigma) \gamma), & \text { if } \frac{\pi}{2} \leq \theta \leq \pi, \\ \cos (\sigma \gamma) \cos ((\theta-\pi-\rho) \gamma), & \text { if } \pi \leq \theta \leq \frac{3 \pi}{2}, \\ \cos \left(\left(\frac{\pi}{2}-\rho\right) \gamma\right) \cos \left(\left(\theta-\frac{3 \pi}{2}-\sigma\right) \gamma\right), & \text { if } \frac{3 \pi}{2} \leq \theta \leq 2 \pi,\end{cases}
$$

and the constants

$$
\gamma=0.1, \quad \rho=\pi / 4, \quad \sigma=-14.9225565104455152, \quad a_{1}=161.4476387975881, \quad a_{2}=1 .
$$

The solution $u$ is barely in $H^{1}(\Omega)$. Indeed, $u \in H^{1+\gamma}(\Omega)$.

Denote the energy error nrgErr $=\left\|u-u_{h}\right\|$. The efficiency index is defined as

$$
\text { effId } x=\frac{\eta}{\operatorname{nrgErr}}
$$

for any specified a posteriori error estimator $\eta$. In the following, we use $\eta_{\mathrm{rec}}^{R T^{0}}$ and $\eta_{\mathrm{rec}}^{B D M^{1}}$ to denote recovery-based error estimator from linear and quadratic recovery procedure,

Table 3: Numerical result for $\eta_{\text {rec }}$ at selected points (Example 6.2).

(a) Recovery-based error estimator $\eta_{\mathrm{rec}}^{R T^{0}}$

\begin{tabular}{||c|c|c|c||}
\hline \# Nodes & nrgErr & $\eta_{\text {rec }}^{R T^{0}}$ & effIdx \\
\hline 289 & 0.6607 & 3.6786 & 5.5674 \\
400 & 0.2247 & 0.8664 & 3.8555 \\
773 & 0.1264 & 0.1692 & 1.3387 \\
1,328 & 0.0829 & 0.1089 & 1.3132 \\
5,373 & 0.0383 & 0.0499 & 1.3001 \\
22,729 & 0.0187 & 0.0242 & 1.2955 \\
92,425 & 0.0092 & 0.0120 & 1.2964 \\
311,183 & 0.0050 & 0.0065 & 1.2958 \\
\hline
\end{tabular}

(b) Recovery-based error estimator $\eta_{\text {rec }}^{B D M^{1}}$

\begin{tabular}{||c|c|c|c||}
\hline \# Nodes & nrgErr & $\eta_{\text {rec }}^{B D M^{1}}$ & effIdx \\
\hline 257 & 0.0698 & 0.2347 & 3.3604 \\
522 & 0.0387 & 0.0525 & 1.3562 \\
807 & 0.0218 & 0.0281 & 1.2906 \\
1,606 & $9.6403 e-3$ & 0.0126 & 1.3043 \\
6,501 & $2.2262 e-3$ & $2.9475 e-3$ & 1.3240 \\
25,237 & $5.6925 e-4$ & $7.6201 e-4$ & 1.3386 \\
103,343 & $1.3908 e-4$ & $1.8718 e-4$ & 1.3458 \\
403,804 & $3.5570 e-5$ & $4.7850 e-5$ & 1.3452 \\
\hline
\end{tabular}




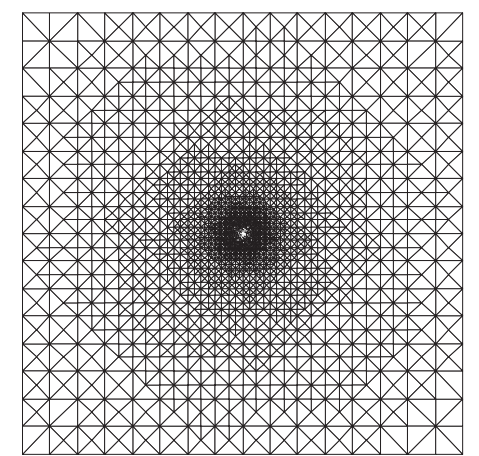

(a) Grid generated by $\eta_{\mathrm{rec}}^{R T^{0}}$ with $N=8291$

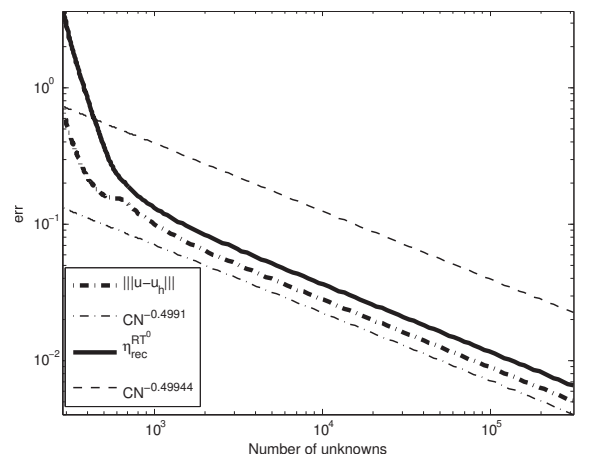

(b) Energy error v.s. $\eta_{\text {rec }}^{R T^{0}}$

Figure 6: Grid and the error history for linear flux recovery-based error estimator $\eta_{\mathrm{rec}}^{R T^{0}}$ (Example 6.2).

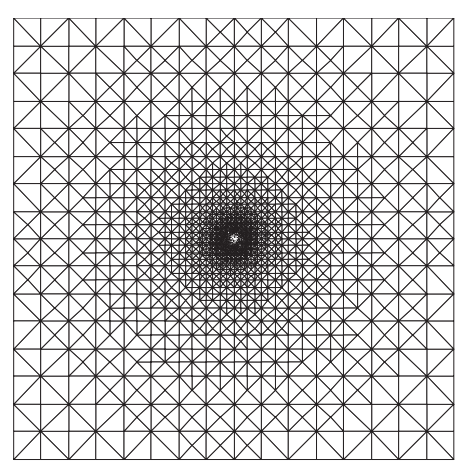

(a) Grid generated by $\eta_{\text {rec }}^{B D M^{1}}$ with $N=8101$

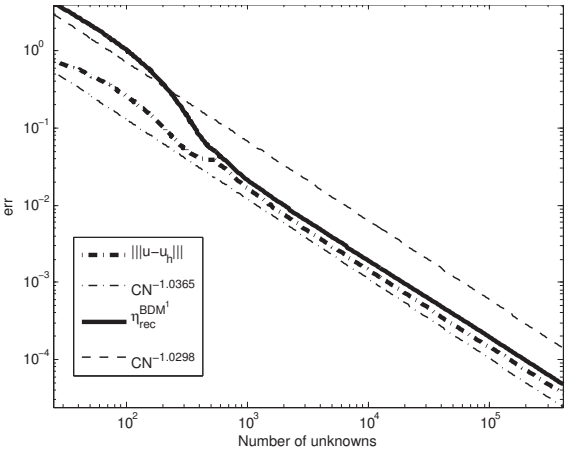

(b) Energy error v.s. $\eta_{\text {rec }}^{B D M^{1}}$

Figure 7: Grid and the error history for quadratic flux recovery-based error estimator $\eta_{\mathrm{rec}}^{B D M^{1}}$ (Example 6.2).

respectively. We select a few number of nodes from the refinement history to show the numerical results for both linear and quadratic recovery in Table 3. Plots of the errors for the whole refinement history are also displayed in Figs. 6 and 7 for an easier comparison. From Table 3, we see that $\eta_{\mathrm{rec}}^{R T^{0}}$ and $\eta_{\mathrm{rec}}^{B D M^{1}}$ are always upper bounds of the energy error.

Table 4: Iteration steps and CPU cost for solving the linear system discretized from linear and quadratic FVMs with $\mathcal{V}$-cycle preconditioned Krylov subspace methods (Example 6.2).

(a) $\mathcal{V}$-cycle preconditioned CG method for linear FVM equation

\begin{tabular}{||c|c|c|c||}
\hline \# Nodes & \# DOF & iterStep & time (s) \\
\hline 1,328 & 1328 & 9 & 0.0822 \\
5,373 & 5373 & 10 & 0.1920 \\
22,729 & 22729 & 11 & 0.4936 \\
92,425 & 92425 & 10 & 1.4792 \\
311,183 & 311183 & 11 & 4.7485 \\
\hline
\end{tabular}

(b) $\mathcal{V}$-cycle preconditioned GMRES method for quadratic FVM equation

\begin{tabular}{||c|c|c|c||}
\hline \# Nodes & \# DOF & iterStep & time (s) \\
\hline 1,606 & 6,397 & 10 & 0.1453 \\
6,501 & 25,949 & 10 & 0.4572 \\
25,237 & 100,841 & 10 & 1.3717 \\
103,343 & 413,165 & 10 & 5.5513 \\
403,804 & $1,614,809$ & 9 & 24.812 \\
\hline
\end{tabular}


We see from Fig. 6 and Fig. 7, the error decrease very fast in the first few steps, and then achieves the optimal convergence rate.

Example 6.3. Steady state flow of fluid through porous medium In this example, we will consider the second-order elliptic equation (1.1) with $f=0$ and $\Omega=(0,1)^{2}$. The diffusion efficient $K=0.001 I$ in the region $(1 / 4,3 / 4) \times(1 / 4,3 / 4)$ and $K=I$ elsewhere. The boundary conditions are given as:

$$
\begin{aligned}
& u=0, \quad \text { on } \Gamma_{D}=\{(1, y): y \in[0,1]\}, \\
& \boldsymbol{K} \nabla u \cdot \boldsymbol{n}= \begin{cases}1, & \text { on } \Gamma_{N}^{i n}=\left\{(0, y): y \in\left[0, \frac{1}{2}\right)\right\}, \\
0, & \text { on } \partial \Omega-\left(\Gamma_{D} \cap \Gamma_{N}^{i n}\right) .\end{cases}
\end{aligned}
$$

See Fig. 8 for the initial computation grid.

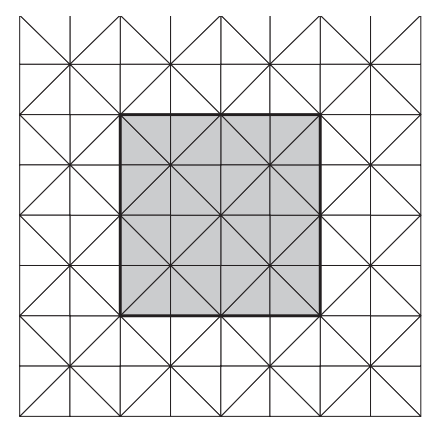

Figure 8: Initial grid for fluid flow problem.

Since it is not clear what is the exact solution of this problem, we will compare our recovered flux with flux calculated from mixed finite element. In Table 5, $\mathrm{L} 2 \mathrm{ErrRT} 0=\left\|\sigma^{R T^{0}}-\sigma_{\text {rec }}^{R T^{0}}\right\|$ and L2ErrBDM1 $=\left\|\sigma^{B D M^{1}}-\sigma_{\text {rec }}^{B D M^{1}}\right\|$ denotes the $L^{2}$ error between the recovered flux and flux calculated from associate mixed FEM, respectively.

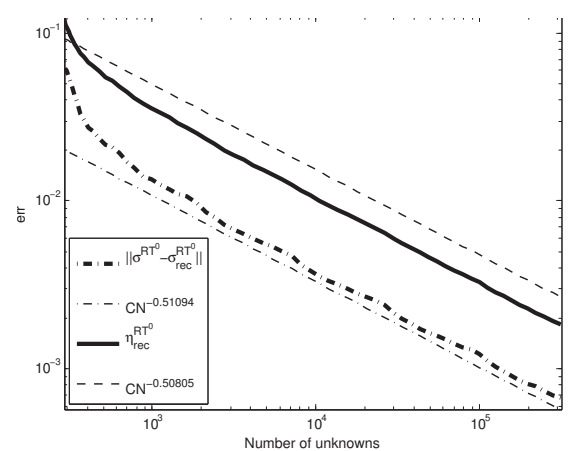

(a) Energy error v.s. $\eta_{\text {rec }}^{R T^{0}}$

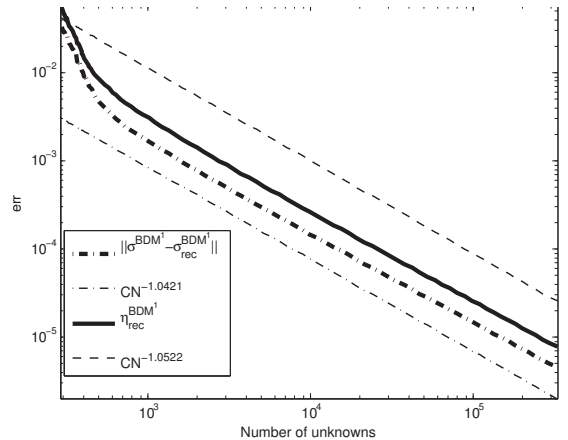

(b) Energy error v.s. $\eta_{\text {rec }}^{B D M^{1}}$

Figure 9: The error history for linear and quadratic flux recovery-based error estimator $\eta_{\mathrm{rec}}^{B D M^{1}}$ (Example 6.3). 
Table 5: Numerical result for $\eta_{\text {rec }}$ at selected points (Example 6.3).

(a) Recovery-based error estimator $\eta_{\text {rec }}^{R T^{0}}$

(b) Recovery-based error estimator $\eta_{\text {rec }}^{B D M^{1}}$

\begin{tabular}{||c|c|c||}
\hline \# Nodes & L2ErrRT0 & $\eta_{\mathrm{rec}}^{R T^{0}}$ \\
\hline 289 & 0.0681 & 0.1254 \\
634 & 0.0194 & 0.0485 \\
1,279 & 0.0117 & 0.0314 \\
5,483 & $5.4042 e-3$ & 0.0144 \\
20,541 & $2.7084 e-3$ & $7.3358 e-3$ \\
80,141 & $1.3588 e-3$ & $3.6859 e-3$ \\
315,935 & $6.8042 e-4$ & $1.8489 e-3$ \\
\hline
\end{tabular}

\begin{tabular}{||c|c|c||}
\hline \# Nodes & L2ErrBDM1 & $\eta_{\mathrm{rec}}^{B D M^{1}}$ \\
\hline 289 & 0.0310 & 0.0564 \\
682 & $2.7597 e-3$ & $5.0563 e-3$ \\
1,465 & $1.0945 e-3$ & $1.9942 e-3$ \\
5,754 & $2.6080 e-4$ & $4.6624 e-4$ \\
21,562 & $6.8870 e-5$ & $1.2098 e-4$ \\
80,140 & $1.8571 e-5$ & $3.2271 e-5$ \\
328,540 & $4.5832 e-6$ & $7.9345 e-6$ \\
\hline
\end{tabular}

Table 6: Iteration steps and CPU cost for solving the linear system discretized from linear and quadratic FVMs with $\mathcal{V}$-cycle preconditioned Krylov subspace methods (Example 6.3).

(a) $\mathcal{V}$-cycle preconditioned CG method for linear FVM equation

\begin{tabular}{||c|c|c|c||}
\hline \# Nodes & \# DOF & iterStep & time (s) \\
\hline 1,279 & 1,279 & 9 & 0.0216 \\
5,483 & 5,483 & 8 & 0.0653 \\
20,541 & 20,541 & 8 & 0.2313 \\
80,141 & 80,141 & 8 & 0.8908 \\
315,935 & 315,935 & 8 & 4.0155 \\
\hline
\end{tabular}

(b) $\mathcal{V}$-cycle preconditioned GMRES method for quadratic FVM equation

\begin{tabular}{||c|c|c|c||}
\hline \# Nodes & \# DOF & iterStep & time (s) \\
\hline 1,465 & 5,774 & 8 & 0.0541 \\
5,754 & 22,850 & 7 & 0.2084 \\
21,562 & 85,924 & 7 & 0.7374 \\
80,140 & 319,916 & 6 & 2.6393 \\
328,540 & $1,312,491$ & 6 & 13.304 \\
\hline
\end{tabular}

As can be clearly seen from Table 5 and Fig. 9, the approximation of mixed FEM and the flux recovery method are comparable in accuracy. Also, the adaptive procedure captures the singularity of the solution very well.

\section{Acknowledgments}

The research of the first author was supported by NSF Grant DMS-1115961 and in part by Department of Energy prime award \#DE-SC0006903. The work of the second author was supported by 2010-2012 China Scholarship Council (CSC).

\section{References}

[1] Y. ACHDOU, C. BERNARDI, AND F. COQUEL, A priori and a posteriori analysis of finite volume discretizations of Darcys equations, Numer. Math., 96 (2003), pp. 17-42.

[2] M. Afif, A. Bergam, Z. MghaZli, AND R. VerfürTh, A posteriori estimators for the finite volume discretization of an elliptic problem, Numer. Algorithms, 34 (2003), pp. 127-136.

[3] A. Agouzal AND F. Oudin, A posteriori error estimator for finite volume methods, Math. Comput., 110 (2000), pp. 239-250. 
[4] M. AINSWORTH, A posteriori error estimation for lowest order Raviart-Thomas mixed finite elements, SIAM J. Sci. Comput., 30 (2007/08), pp. 189-204.

[5] M. AinswORTH AND J. ODEN, A posteriori error estimation in finite element analysis, Wiley-Interscience, 2000.

[6] M. AINSWORTH AND R. RANKIN, Fully computable bounds for the error in nonconforming finite element aoximations of arbitrary order on triangular elements, SIAM J. Numer. Anal., 46 (2008), pp. 3207-3232.

[7] R. BANK AND D. RosE, Some error estimates for the box method, SIAM J. Numer. Anal., 24 (1987), pp. 777-787.

[8] D. BRAESS, Finite Elements: Theory, Fast Solvers, and Aications in Solid Mechanics, 2nd ed., Cambridge University Press, Cambridge, 2001.

[9] D. BRAESS AND J. SCHOBERL, Equilibrated residual error estimator for edge elements, Math. Comput., 77 (2008), pp. 651-672.

[10] Z. CAI, J. DOUgLAS JR, AND M. PARK, Development and analysis of higher order finite volume methods over rectangles for elliptic equations, Adv. Comput. Math., 19 (2003), pp. 3-33.

[11] Z. CAI AND S. ZHANG, Recovery-based error estimator for interface problems: conforming linear elements, SIAM J. Numer. Anal., 47 (2009), pp. 2132-2156.

[12] C. CARSTENSEN, R. LAZAROV, AND S. TOMOV, Explicit and averaging a posteriori error estimates for adaptive finite volume methods, SIAM J. Numer. Anal., 42 (2005), pp. 2496-2521.

[13] L. CHEN, iFEM: An Integrated Finite Element Methods Package in Matlab, University of California at Irvine, Technical Report, 2009.

[14] L. CHEN, A new class of high order finite volume methods for second order elliptic equations, SIAM J. Numer. Anal., 47 (2010), pp. 4021-4043.

[15] S. CHOU, S. HE, AND W. LIN, Conservative flux recovery from the Q1 conforming finite element method on quadrilateral grids, Numer. Methods Partial Differential Equations, 20 (2004), pp. $104-127$.

[16] S. CHOU, D. KWAK, AND K. KIM, Flux recovery from primal hybrid finite element methods, SIAM J. Numer. Anal., 40 (2003), pp. 403-415.

[17] W. DÖRFLER, A convergent adaptive algorithm for Poisson's equation, SIAM J. Numer. Anal., 33 (1996), pp. 1106-112.

[18] C. ERATH AND D. PRAETORIUS, A posteriori error estimate and adaptive mesh-refinement for the cell-centered finite volume method for elliptic boundary value problems, SIAM J. Numer. Anal., 47 (2008), pp. 109-135.

[19] O. KARAKASHIAN AND F. PASCAL, A posteriori error estimates for a discontinuous Galerkin aoximation of second-order elliptic problems, SIAM J. Numer. Anal., 41 (2003), pp. 2374-2399.

[20] R. KellogG, On the Poisson equation with intersecting interfaces, Aicable Anal., 4 (1974), pp. 101-129.

[21] R. Li, Z. CHEN, AND W. WU, Generalized Difference Methods for Differential Equations, Marcel Dekker, New York, 2000.

[22] F. LIEBAU, The finite volume element method with quadratic basis functions, Computing, 57 (1996), pp. 281-299.

[23] P. MORIN, R. NOCHETTO, AND K. SIEBERT, Data oscillation and convergence of adaptive FEM, SIAM J. Numer. Anal., 38 (2001), pp. 466-488.

[24] S. NICAISE, A posteriori error estimations of some cell-centered finite volume methods, SIAM J. Numer. Anal., 43 (2005), pp. 1481-1503.

[25] Y. LI, S. SHU, Y. XU, AND Q. ZOU, Multilevel preconditioning for the finite volume method, Math. Comput., 81 (2012), pp. 1399-1428. 
[26] Y. LI AND R. LI, Generalized difference methods on arbitrary quadrilateral networks, J. Comput. Math., 17 (1999), pp. 653-672.

[27] S. SHU, H. YU, Y. HUANG AND C. NIE, A symmetric finite volume element scheme on quadrilateral grids and superconvergence, J. Numer. Anal. Model., 1 (2006), pp. 1-18.

[28] S. LIANG, X. MA AND A. ZHOU, Finite volume methods for eigenvalue problems, BIT, 41 (2000), pp. 345-363.

[29] S. LIANG, X. MA, AND A. ZHOU, A symmetric finite volume scheme for selfadjoint elliptic problems, J. Comput. A. Math., 147 (2002), pp. 121-136.

[30] X. MA, S. SHU AND A. ZHOU, Symmetric finite volume discretization for parabolic problems, Comput. Methods A. Mech. Eng., 192 (2003), pp. 4467-4485.

[31] J. JiAnG, S. SHU AND Y. HUANG, Symmetric finite volume element method in ICF, J. System Simulation.

[32] W. Prager AND J. SYNGe, Aoximations in elasticity based on the concept of function space, Quart. A. Math., 5 (1947), pp. 241-269.

[33] T. RusSELl AND M. WHEELER, Finite element and finite difference methods for continuous flows in porous media, The Mathematics of Reservoir Simulation, 1 (1983), pp. 35-106.

[34] E. STEIN AND M. RÜTER, Finite element methods for elasticity with error-controlled discretization and model adaptivity, Encyclopedia of Computational Mechanics, 2007.

[35] E. SÜLI, Convergence of finite volume schemes for Poisson's equation on nonuniform meshes, SIAM J. Numer. Anal., 28 (1991), pp. 1419-1430.

[36] J. SYNGE AND W. RHEINBOLDT, The hypercircle in mathematical physics, Phys. Today, (1957), pp. 10-45.

[37] S. TOMOV, Adaptive Methods for Finite Volume Aoximations, Thesis (Ph.D.)-Texas A\& M University, 2002.

[38] T. VEJCHODSKỲ, Guaranteed and locally computable a posteriori error estimate, IMA J. Numer. Anal., 26 (2006), pp. 525-540.

[39] R. VERfÜRTH, A Review of a Posteriori Error Estimation and Adaptive Mesh-Refinement Techniques, Wiley-Teubner, Chichester and New York, 1996.

[40] A. VoGel, J. XU, AND G. WitTum, A generalization of the vertex-centered finite volume scheme to arbitrary high order, Comput. Vis. Sci., 13 (2010), pp. 1-8.

[41] J. XU AND Q. ZOU, Analysis of linear and quadratic simplicial finite volume methods for elliptic equations, Numer. Math., 111 (2009), pp. 469-492.

[42] D. Hilhorst AND M. VOHRALÍK, A posteriori error estimates for combined finite volume-finite element discretizations of reactive transport equations on nonmatching grids, Comput. Methods A. Mech. Eng., 200 (2011), pp. 597-613. 